\title{
Efficacy and Safety of Intra-articular Injections of Hyaluronic Acid Combined With Polydeoxyribonucleotide in the Treatment of Knee Osteoarthritis
}

Seihee Yoon, MD, Jung Joong Kang, MD, Jungin Kim, MD, Seunghun Park, MD, Jong Moon Kim, MD, PhD

Department of Rehabilitation Medicine, Konkuk University Chungju Hospital, Konkuk University School of Medicine, Chungju, Korea

Objective To assess the clinical efficacy and safety of intra-articular injection of hyaluronic acid (HA) combined with polydeoxyribonucleotide (PDRN) in patients with knee osteoarthritis in comparison with that of HA alone. Methods The current single-center, prospective, randomized, double-blind, controlled study was conducted in 36 patients with knee osteoarthritis at our medical institution. All the eligible patients $(n=30)$ were equally assigned to two treatment arms (trial group 'HA+PDRN' and control group 'HA'). For efficacy assessment, the patients were evaluated for the visual analogue scale (VAS) scores, the Western Ontario and McMaster Universities Osteoarthritis Index (WOMAC) and the Knee Society Scores (KSS), all of which served as efficacy outcome measures. We monitored time-dependent changes in efficacy outcome measures at baseline and 1, 3 and 6 months. Subsequently, we compared differences in changes in efficacy outcome measures at 6 months from baseline between the two groups. Moreover, we assessed the safety based on the treatment-emergent adverse events (TEAEs), adverse drug reactions (ADRs) and any other complications serving as safety outcome measures. Results There were significant differences in changes in the VAS scores, the WOMAC scores in all domains, except 'Stiffness', the total WOMAC scores, and the KSS scores in all the domains at 6 months from baseline between the two groups $(\mathrm{p}<0.05)$. In our series, there were no TEAEs, ADRs, and any other complications.

Conclusion Intra-articular injections of HA combined with PDRN can also be considered in the treatment of knee osteoarthritis. However, further large-scale and multi-center studies are required to demonstrate the potential of the proposed combination.

Keywords Knee osteoarthritis, Intra-articular injections, Hyaluronic acid, Polydeoxyribonucleotides

Department of Rehabilitation Medicine, Konkuk University Chungju Hospital, Konkuk University School of Medicine, 82 Gugwon-daero, Chungju 27376, Korea. Tel: +82-43-840-8890, Fax: +82-43-840-8968, E-mail: rmjmk@kku.ac.kr

ORCID: Seihee Yoon (http://orcid.org/0000-0002-2153-666X); Jung Joong Kang (http://orcid.org/0000-0003-2726-7824); Jungin Kim (http://orcid. org/0000-0003-2999-3264); Seunghun Park (http://orcid.org/0000-0002-3008-5768); Jong Moon Kim (http://orcid.org/0000-0003-0702-4243).

(c) This is an open-access article distributed under the terms of the Creative Commons Attribution Non-Commercial License (http://creativecommons.org/ licenses/by-nc/4.0) which permits unrestricted noncommercial use, distribution, and reproduction in any medium, provided the original work is properly cited. Copyright $\odot 2019$ by Korean Academy of Rehabilitation Medicine 


\section{INTRODUCTION}

Osteoarthritis (OA) is the most common form of articular disease affecting mainly hips, knees, hands and feet [1]. Patients with OA are vulnerable to morbidity, disability and functional deficits [2]. The number of patients with OA is estimated at 151 million worldwide. Symptomatic knee OA has emerged as the most common form of OA in the elderly aged 65 years or older [3-5]. Furthermore, OA is closely associated with the increased burden on the healthcare resources [6].

There are various types of treatment modalities for patients with OA. Of these, intra-articular injections of corticosteroids, analgesics, anti-inflammatory drugs, polymerized collagen, and anti-cytokine drugs or hyaluronic acid (HA), termed as viscosupplementation, have also been attempted in an effort to enhance topical effects and to reduce systemic adverse effects [7].

HA is a slow-acting treatment agent for symptomatic $\mathrm{OA}$, and its moderate efficacy against knee OA pain has been well described in the literature [8]. According to a meta-analysis of previously published studies in this series, HA exhibited excellent efficacy in relieving pain as compared with the placebo-controlled group $[9,10]$. Moreover, recently, efforts have been made to increase the efficacy of HA with decreased cytotoxicity $[11,12]$. Furthermore, its combination with anti-inflammatory agents has also been studied [13].

To date, DNA polymeric molecules, such as polydeoxyribonucleotides (PDRN) and polynucleotides (PN) have been used to treat patients with knee OA. Consequently, Zazgyva et al. [14] showed that PN injections were effective in treating patients with symptomatic knee OA. Moreover, two clinical trials have assessed the efficacy of $\mathrm{PN}$, and that of PN combined with HA as compared with HA alone $[15,16]$. PDRN is effective in treating patients with plantar fasciitis, lumbosacral radiculopathy, and supraspinatus tendinopathy. In addition, it has been reported to be effective against rheumatoid arthritis in animal models [17-20]. Unlike NSAIDs, PDRN exerts antiinflammatory actions without metabolic adverse effects, which makes it a possible alternative to steroids for the treatment of musculoskeletal disorders. According to a review of literature, there is a paucity of studies comparing the efficacy of PDRN combined with HA with that of $\mathrm{HA}$ alone in patients with knee OA.
Based on the existing background, we conducted the present study to assess the efficacy and safety of intraarticular injection of HA combined with PDRN as compared with that of HA alone in patients with knee OA.

\section{MATERIALS AND METHODS}

\section{Study patients and setting}

For the current single-center, prospective, randomized, double-blind, and controlled study, inclusion criteria were as follows: (1) men or women aged 50 years or older; (2) patients with a persistent presence of knee joint pain for more than 2 months; and (3) patients with radiological findings, such as the formation of osteophytes, narrowing of the joint gap and flattening of the femoral head that are indicative of the Kellgren-Lawrence (KL) grade II or III. Exclusion criteria for the current study were as follows: (1) patients with a past history of any treatments that may affect the results of the current study (e.g., surgeries including knee arthroplasty, pharmacological treatments, HA injections, physical therapy, and exercise); (2) patients with other underlying medical conditions that may affect the results of the current study; (3) patients who have received steroid injections; (4) patients with a history of trauma or fracture of the knee joint; (5) patients with hypersensitivity to study treatments; and (6) patients who were deemed to be ineligible for study participation according to our judgment.

The current study was approved by the Institutional Review Board of Konkuk University Chungju Hospital (No. 2017-06-016). All the patients submitted a written informed consent for study participation. The current study was conducted in accordance with the Declaration of Helsinki.

\section{The rationale of sample size estimation}

The sample size was estimated based on a decrease in changes in the VAS scores. This is accompanied by the assumption that there would be a lack of interaction between the two treatments. Consequently, we calculated the sample size through comparison of 2 means with a standard deviation of 90 [21]. Considering a significance level of $5 \%$, a statistical power of $90 \%$ and a drop-out rate of $20 \%$, we estimated the sample size of 15 per group. 


\section{Treatment protocol}

A total of 30 enrolled patients were randomized to two treatment arms using a permuted block design. The patients of the trial group $(n=15)$ and the control group $(\mathrm{n}=15)$ received an intra-articular injection of HA (Hylon Plus inj.; Aju Pharm Co. Ltd., Seoul, Korea) combined with PDRN (Placentex Inj.; Mastelli srl, Sanremo, Italy) and HA alone, respectively, by employing a 21-gauge needle. The treatment was done once a week (a total of three times).

For intra-articular injections of study treatments, the knee region was completely disinfected with Betadine Solution and then totally extended. Next, the patella was pushed upward and then shifted to the lateral side; this was followed by the transverse insertion of the needle between the articular surface and the patellofemoral joint on the midpoint of the patella. Intra-articular injections of study treatments were performed via a superolateral patellar approach by a highly-skilled physician, under aseptic conditions by following the standard guidelines for intra-articular injection. In the trial group, intra-articular injection of PDRN was followed by that of HA without changing the needles. In both the groups, we used a blind intra-articular injection of study treatments. Prior to this, arthrocentesis was performed to prevent the knee effusion as well as to confirm the proper placement of the needle in the knee joint [22].

For analysis of the synovial fluid, an excess of the fluid was removed and the aliquot was taken at an approximate volume of $6 \mathrm{~mL}$ under sterile conditions. No abnormalities in the clarity, color, and viscosity of the synovial fluid were observed.

\section{Patient evaluation and criteria}

The patients were evaluated by coauthors who were not involved in injections of study treatments. The patients were assessed for efficacy and safety at baseline and followed up at 1, 3 and 6 months. The patients were evaluated for the pain level using the VAS score. Moreover, the patients were asked to respond to a self-administered questionnaire using the Western Ontario and McMaster Universities Osteoarthritis Index (WOMAC) and the Knee Society Score (KSS).

\section{Evaluation tools \\ VAS}

The VAS is composed of a straight line with the endpoints indicating extreme limits ('no pain at all' and 'pain as bad as it could be'). For the assessment of pain, the patients were asked to mark their pain level on the line between the two endpoints. Accordingly, their pain level was defined as the distance extending from the point indicating 'no pain at all' and the mark [23].

\section{WOMAC}

The WOMAC is a self-administered questionnaire that is composed of 24 questions categorized into three subscales (pain, stiffness, and physical function). In the current study, we used 5-point Likert scale format: 'none' (0), 'mild' (1), 'moderate' (2), 'severe' (3), and 'extreme' (4). Scores for each subscale were determined by summing the component item scores for each subscale-possible score range: pain (0-20), stiffness (0-8), and physical function (0-68). The final total scores (possible score range, 0-96) were determined by summing the scores for each subscale [24]. In the current study, we used the Korean version of WOMAC the reliability and validity of which were described in the literature [25] (Appendix 1).

\section{KSS}

The KSS combines subjective and objective information, and it differentiates the knee score (pain, stability, and $\mathrm{ROM}$ ) from the patient's functional one (ability to walk and climb up and down stairs) [26]. In the current study, however, we used the Korean version of the 2011 KSS, which consisted of four subscales: (A) objective knee scores ( 7 items, 100 points); (B) symptom scores (3 items, 25 points); (C) patient satisfaction scores (5 items, 40 points); and (D) functional activity scores (19 items, 100 points). The reliability and validity of the Korean version of the 2011 KSS were described in the literature $[27,28]$ (Appendix 2).

\section{Efficacy outcome measures and assessment}

For efficacy assessment, the patients were evaluated for the VAS scores, the WOMAC, and the KSS, all of which served as efficacy outcome measures. We monitored time-dependent changes in efficacy outcome measures at baseline and 1, 3 and 6 months. Subsequently, we compared differences in changes in efficacy outcome 
measures at 6 months from baseline between the two groups. Moreover, we performed an intent-to-treat (ITT) analysis.

The ITT set comprises of all the patients who were given the randomization number after being enrolled in the current study. But we excluded the following patients: (1) patients who were enrolled in the current study even though they did not meet inclusion/exclusion criteria at the screening visit; (2) patients who were enrolled in the current study but did not undergo treatment; and (3) patients who were enrolled in the current study but did not undergo efficacy analysis.

\section{Safety outcome measures and assessment}

For safety assessment, we examined whether the patients presented with the treatment-emergent adverse events (TEAEs), adverse drug reactions (ADRs) and any other complications serving as safety outcome measures.

\section{Statistical analysis}

Data are expressed as mean \pm standard deviation. Statistical analysis was done using the SPSS version 18.0 for Windows (SPSS Inc., Chicago, IL, USA). We compared differences in changes in efficacy outcome measures at 6

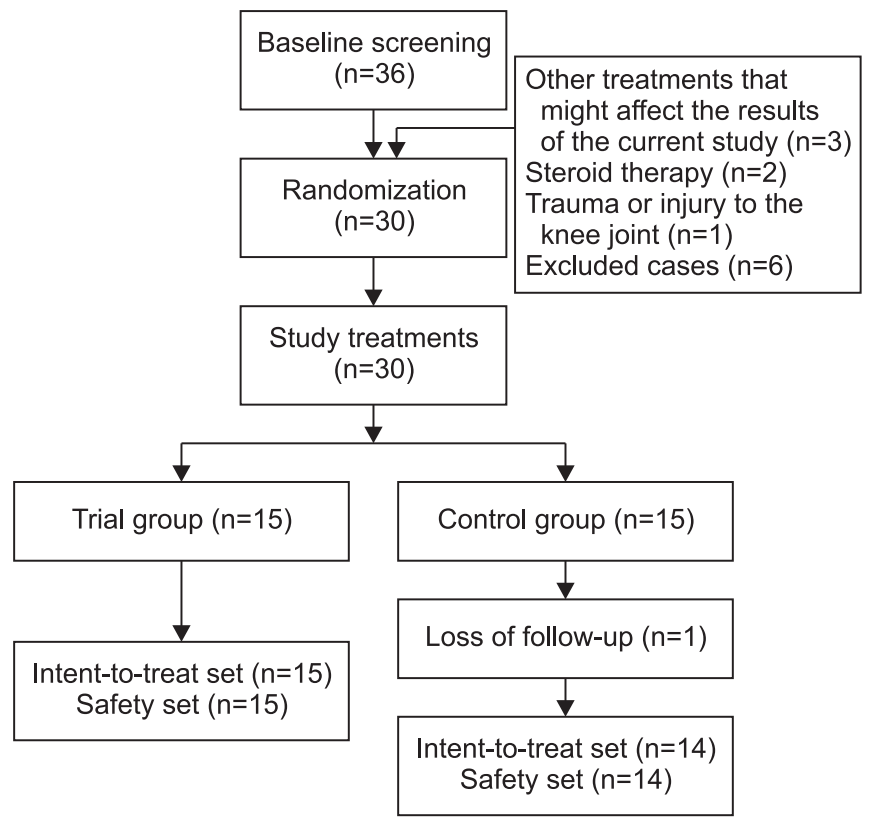

Fig. 1. The study flow chart. A total of 30 enrolled patients were randomized to two treatment arms. With the exclusion of one case of lost-to-follow-up in the control group, the remaining 29 patients completed the entire study. months from baseline between the two groups using the Student t-test. A p-value of $<0.05$ was considered statistically significant.

\section{RESULTS}

\section{Baseline characteristics of the patients}

Of the 36 recruited patients, a total of 30 patients met inclusion/exclusion criteria and were randomized to two treatment arms. However, there was one case of lost-tofollow-up in the control group. Consequently, 15 patients of the trial group and 14 of the control group completed the entire study. The study flow chart is shown in Fig. 1.

Our clinical series of the patients consisted of 13 men and 16 women with a mean age of $65.10 \pm 4.53$ years. Patients had a height of $160.14 \pm 8.73 \mathrm{~cm}$, weight of $65.45 \pm 10.78 \mathrm{~kg}$ and BMI of $25.45 \pm 3.15 \mathrm{~kg} / \mathrm{m}^{2}$. There were 20 and 9 patients with the K-L grade II and III on radiological findings, respectively. Baseline characteristics of the patients of each group are represented in Table 1.

Time-dependent changes in efficacy outcome measures and efficacy outcomes

Time-dependent changes in efficacy outcome measures in each group are shown in Table 2.

There were significant differences in changes in the VAS scores at 6 months from baseline between the two groups $(\mathrm{p}=0.0001)$. Also, significant differences in changes in the WOMAC scores in all domains, except 'stiffness', at

Table 1. Baseline characteristics of the patients in each group

\begin{tabular}{lccc}
\hline & $\begin{array}{c}\text { Trial group } \\
(\mathbf{n}=15)\end{array}$ & $\begin{array}{c}\text { Control group } \\
(\mathbf{n}=\mathbf{1 4})\end{array}$ & p-value \\
\hline Age $(\mathrm{yr})$ & $65.33 \pm 4.78$ & $64.86 \pm 4.45$ & $>0.05$ \\
Sex & & & \\
$\quad$ Male & 7 & 6 & \\
Female & 8 & 8 & \\
Height $(\mathrm{cm})$ & $160.40 \pm 8.98$ & $159.86 \pm 8.78$ & $>0.05$ \\
Weight $(\mathrm{kg})$ & $64.93 \pm 10.79$ & $66.00 \pm 11.14$ & $>0.05$ \\
BMI $\left(\mathrm{kg} / \mathrm{m}^{2}\right)$ & $25.18 \pm 3.19$ & $25.74 \pm 3.20$ & $>0.05$ \\
K-L grade & & & - \\
I & 11 & 9 & \\
$\quad$ II & 4 & 5 & \\
\hline
\end{tabular}

Values are presented as mean \pm standard deviation. BMI, body mass index; K-L, Kellgren-Lawrence. 


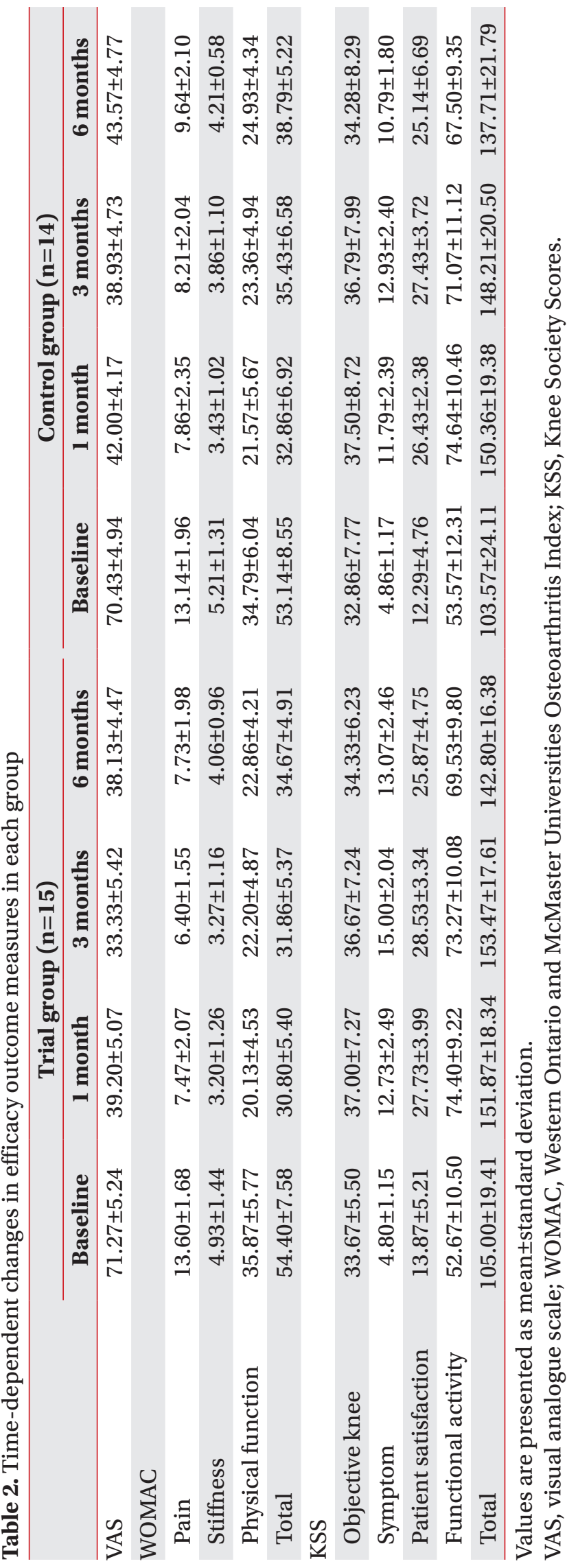




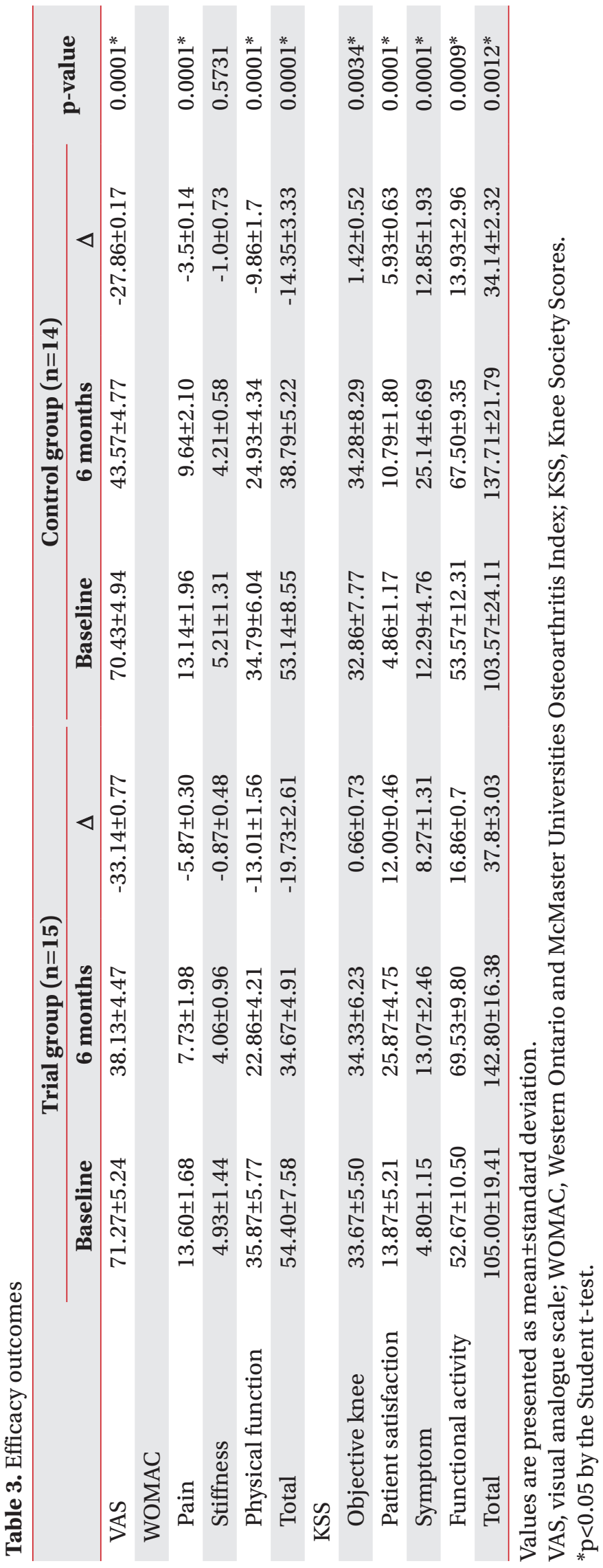


6 months from baseline between the two groups were observed ( $\mathrm{p}=0.0001$ for pain, $\mathrm{p}=0.5731$ for stiffness, and $\mathrm{p}=0.0001$ for physical function). Moreover, there were significant differences in changes in the total WOMAC scores at 6 months from baseline between the two groups $(\mathrm{p}=0.0001)$.

There were significant differences in changes in the KSS scores in all the domains at 6 months from baseline between the two groups ( $p=0.0034$ for objective knee scores, $\mathrm{p}=0.0001$ for patient satisfaction scores, $\mathrm{p}=0.0001$ for symptom scores, and $\mathrm{p}=0.0009$ for functional activity scores). Moreover, there were significant differences in changes in the total KSS scores at 6 months from baseline between the two groups $(\mathrm{p}=0.0012)$ (Table 3 ).

\section{Safety outcomes}

In our series, there were no TEAEs, ADRs, and any other complications.

\section{DISCUSSION}

Briefly, our results showed significant improvement in the VAS, WOMAC, except for the 'stiffness', and KSS scores in the trial group as compared with the control group. These results suggest that the degree of efficacy outcomes was significantly higher in the patients receiving HA combined with PDRN as compared with those undergoing HA alone treatment. It can be, therefore, inferred that the combination of PDRN with HA was effective in enhancing the efficacy of HA in the treatment of patients with knee OA. As described earlier, Dallari et al. [16] showed that the combination of PN (one of the DNA polymeric molecules) with HA, enhanced the efficacy of HA in the treatment of knee OA. Taken together, we assume that there is a good level of evidence that intra-articular injection of HA in combination with anti-inflammatory drugs, such as PDRN, can potentially improve pain in patients with knee OA without notable adverse events as compared with HA treatment alone [13].

Until date, there exists little information about the effects of PDRN in enhancing the anti-inflammatory actions of HA. To date, such effects have been suggested in experimental studies rather than clinical ones. Bitto et al. [20] showed that PDRN influenced the levels of cytokines, such as high mobility group box-1 (HMGB-1), tumor necrosis factor- $\alpha$ (TNF- $\alpha$ ), interleukin (IL)-6 and IL-10 in human chondrocytes. In human chondrocytes, IL-10 levels were elevated following the treatment with PDRN. IL10 plays a role in inhibiting the synthesis of pro-inflammatory cytokines in activated macrophages, T-cells and polymorphonuclear leukocytes, reducing the synthesis of matrix metalloproteinases (MMPs), suppressing the expression of class II major histocompatibility complex, and lowering the proliferation of Thl lymphocytes. Consequently, it can be inferred that curative effects of PDRN might also arise, at least in part, from up-regulation of IL10 which is involved in down-regulating the up-regulated expression of the early and late inflammatory factors. Moreover, according to Gennero et al. [29], following the treatment with PDRN, chondrocytes demonstrated physiological deposition of extracellular matrices, such as aggrecan and type II collagen, accompanied by inhibition of proteoglycan degradation in cartilage explants. In addition, a decrease in the levels of MMP-2 and -9 after the treatment with PDRN was reported. Overall, these findings suggest that the effects of PDRN in relieving pain might arise from anti-inflammatory actions.

In the current study, we also found that an intra-articular injection of PDRN combined with HA was effective in enhancing the efficacy of HA in improving the functions of the knee joint; the WOMAC physical function scores and the KCC functional activity were significantly improved in the trial group as compared with the control group ( $\mathrm{p}=0.0001$ and $\mathrm{p}=0.0009$, respectively). This observation is in agreement with the result of a previously published study showing that PN, one of the DNA polymeric molecules, was effective in improving the KSS and WOMAC scores when combined with HA as compared with HA alone in the treatment of knee OA [16]. Admitting that other treatments, such as pharmacological treatments, physical therapy, exercise, and arthroplasty, are effective in improving the functions of the knee joint, we propose that PDRN combined with HA can be considered an alternative treatment modality in this series.

In conclusion, our results indicate that intra-articular injections of HA combined with PDRN might be useful in enhancing the efficacy of HA alone in the treatment of knee OA. However, our results cannot be generalized as we evaluated a small number of patients who were treated at a single, secondary medical institution. Apparently, we could not completely rule out the possibility of selection bias. In future, large-scale and multi-center studies 
are warranted to establish our results.

\section{CONFLICT OF INTEREST}

No potential conflict of interest relevant to this article was reported.

\section{AUTHOR CONTRIBUTION}

Conceptualization: Kim JM, Yoon S. Methodology: Kim JM, Yoon S. Formal analysis: Kim J, Park S. Project administration: Kang JJ, Yoon S. Visualization: Park S. Writing - original draft: Yoon S, Kang JJ. Writing - review and editing: Kim JM, Yoon S. Approval of final manuscript: all authors.

\section{REFERENCES}

1. Litwic A, Edwards MH, Dennison EM, Cooper C. Epidemiology and burden of osteoarthritis. Br Med Bull 2013;105:185-99.

2. Rosemann T, Laux G, Kuehlein T. Osteoarthritis and functional disability: results of a cross sectional study among primary care patients in Germany. BMC Musculoskelet Disord 2007;8:79.

3. Bhatia D, Bejarano T, Novo M. Current interventions in the management of knee osteoarthritis. J Pharm Bioallied Sci 2013;5:30-8.

4. Lawrence RC, Felson DT, Helmick CG, Arnold LM, Choi H, Deyo RA, et al. Estimates of the prevalence of arthritis and other rheumatic conditions in the United States. Part II. Arthritis Rheum 2008;58:26-35.

5. Felson DT, Lawrence RC, Dieppe PA, Hirsch R, Helmick CG, Jordan JM, et al. Osteoarthritis: new insights. Part 1. The disease and its risk factors. Ann Intern Med 2000;133:635-46.

6. Le TK, Montejano LB, Cao Z, Zhao Y, Ang D. Health care costs in US patients with and without a diagnosis of osteoarthritis. J Pain Res 2012;5:23-30.

7. Iannitti T, Lodi D, Palmieri B. Intra-articular injections for the treatment of osteoarthritis: focus on the clinical use of hyaluronic acid. Drugs R D 2011;11:1327.

8. Chard J, Dieppe P. Update: treatment of osteoarthritis. Arthritis Rheum 2002;47:686-90.

9. Bannuru RR, Natov NS, Obadan IE, Price LL, Schmid
$\mathrm{CH}$, McAlindon TE. Therapeutic trajectory of hyaluronic acid versus corticosteroids in the treatment of knee osteoarthritis: a systematic review and metaanalysis. Arthritis Rheum 2009;61:1704-11.

10. Bellamy N, Campbell J, Robinson V, Gee T, Bourne R, Wells G. Viscosupplementation for the treatment of osteoarthritis of the knee. Cochrane Database Syst Rev 2006;(2):CD005321.

11. Hashizume M, Mihara M. Desirable effect of combination therapy with high molecular weight hyaluronate and NSAIDs on MMP production. Osteoarthritis Cartilage 2009;17:1513-8.

12. Onur TS, Sitron CS, Dang A. Co-administration of hyaluronic acid with local anaesthetics shows lower cytotoxicity than local anaesthetic treatment alone in bovine articular chondrocytes. Bone Joint Res 2013;2: 270-5.

13. Euppayo T, Punyapornwithaya V, Chomdej S, Ongchai S, Nganvongpanit K. Effects of hyaluronic acid combined with anti-inflammatory drugs compared with hyaluronic acid alone, in clinical trials and experiments in osteoarthritis: a systematic review and metaanalysis. BMC Musculoskelet Disord 2017;18:387.

14. Zazgyva A, Gergely I, Russu OM, Roman C, Pop TS. Polynucleotides versus sodium hyaluronate in the local treatment of knee osteoarthritis. Acta Medica Transilvanica 2013;2:260-3.

15. Vanelli R, Costa P, Rossi SM, Benazzo F. Efficacy of intra-articular polynucleotides in the treatment of knee osteoarthritis: a randomized, double-blind clinical trial. Knee Surg Sports Traumatol Arthrosc 2010;18: 901-7.

16. Dallari D, Sabbioni G, Del Piccolo N, Carubbi C, Veronesi F, Torricelli $\mathrm{P}$, et al. Efficacy of intra-articular polynucleotides associated with hyaluronic acid versus hyaluronic acid alone in the treatment of knee osteoarthritis: a randomized, double-blind, controlled clinical trial. Clin J Sport Med 2018 Apr 10 [Epub]. http://doi.org/10.1097/JSM.0000000000000569.

17. Kim JK, Chung JY. Effectiveness of polydeoxyribonucleotide injection versus normal saline injection for treatment of chronic plantar fasciitis: a prospective randomised clinical trial. Int Orthop 2015;39:1329-34.

18. Kang KN, Kim TW, Koh JW, Oh HB, Mun JU, Seo MS, et al. Effect of transforaminal epidural polydeoxyribonucleotide injections on lumbosacral radiculopathy: a 
case report. Medicine (Baltimore) 2017;96:e7174.

19. Yoon YC, Lee DH, Lee MY, Yoon SH. Polydeoxyribonucleotide injection in the treatment of chronic supraspinatus tendinopathy: a case-controlled, retrospective, comparative study with 6-month follow-up. Arch Phys Med Rehabil 2017;98:874-80.

20. Bitto A, Polito F, Irrera N, D'Ascola A, Avenoso A, Nastasi G, et al. Polydeoxyribonucleotide reduces cytokine production and the severity of collagen-induced arthritis by stimulation of adenosine $\mathrm{A}\left({ }_{2} \mathrm{~A}\right)$ receptor. Arthritis Rheum 2011;63:3364-71.

21. Dougados M, Leclaire P, van der Heijde D, Bloch DA, Bellamy N, Altman RD. Response criteria for clinical trials on osteoarthritis of the knee and hip: a report of the Osteoarthritis Research Society International Standing Committee for Clinical Trials response criteria initiative. Osteoarthritis Cartilage 2000;8:395-403.

22. Lee JK, Lee BY, Shin WY, An MJ, Jung KI, Yoon SR. Effect of extracorporeal shockwave therapy versus intraarticular injections of hyaluronic acid for the treatment of knee osteoarthritis. Ann Rehabil Med 2017;41: 828-35.

23. Haefeli M, Elfering A. Pain assessment. Eur Spine J 2006;15 Suppl 1:S17-24.

24. Bellamy N, Buchanan WW, Goldsmith CH, Campbell J, Stitt LW. Validation study of WOMAC: a health status instrument for measuring clinically important patient relevant outcomes to antirheumatic drug therapy in patients with osteoarthritis of the hip or knee. J Rheumatol 1988; 15:1833-40.

25. Bae SC, Lee HS, Yun HR, Kim TH, Yoo DH, Kim SY. Cross-cultural adaptation and validation of Korean Western Ontario and McMaster Universities (WOMAC) and Lequesne osteoarthritis indices for clinical research. Osteoarthritis Cartilage 2001;9:746-50.

26. Insall JN, Dorr LD, Scott RD, Scott WN. Rationale of the Knee Society clinical rating system. Clin Orthop Relat Res 1989;(248):13-4.

27. Scuderi GR, Bourne RB, Noble PC, Benjamin JB, Lonner JH, Scott WN. The new Knee Society Knee Scoring System. Clin Orthop Relat Res 2012;470:3-19.

28. Kim SJ, Basur MS, Park CK, Chong S, Kang YG, Kim $\mathrm{MJ}$, et al. Crosscultural adaptation and validation of the Korean version of the New Knee Society Knee Scoring System. Clin Orthop Relat Res 2017;475:162939.

29. Gennero L, Denysenko T, Calisti GF, Vercelli A, Vercelli CM, Amedeo S, et al. Protective effects of polydeoxyribonucleotides on cartilage degradation in experimental cultures. Cell Biochem Funct 2013;31:21427. 
Appendix 1. Western Ontario and McMaster Universities Osteoarthritis Index (WOMAC)

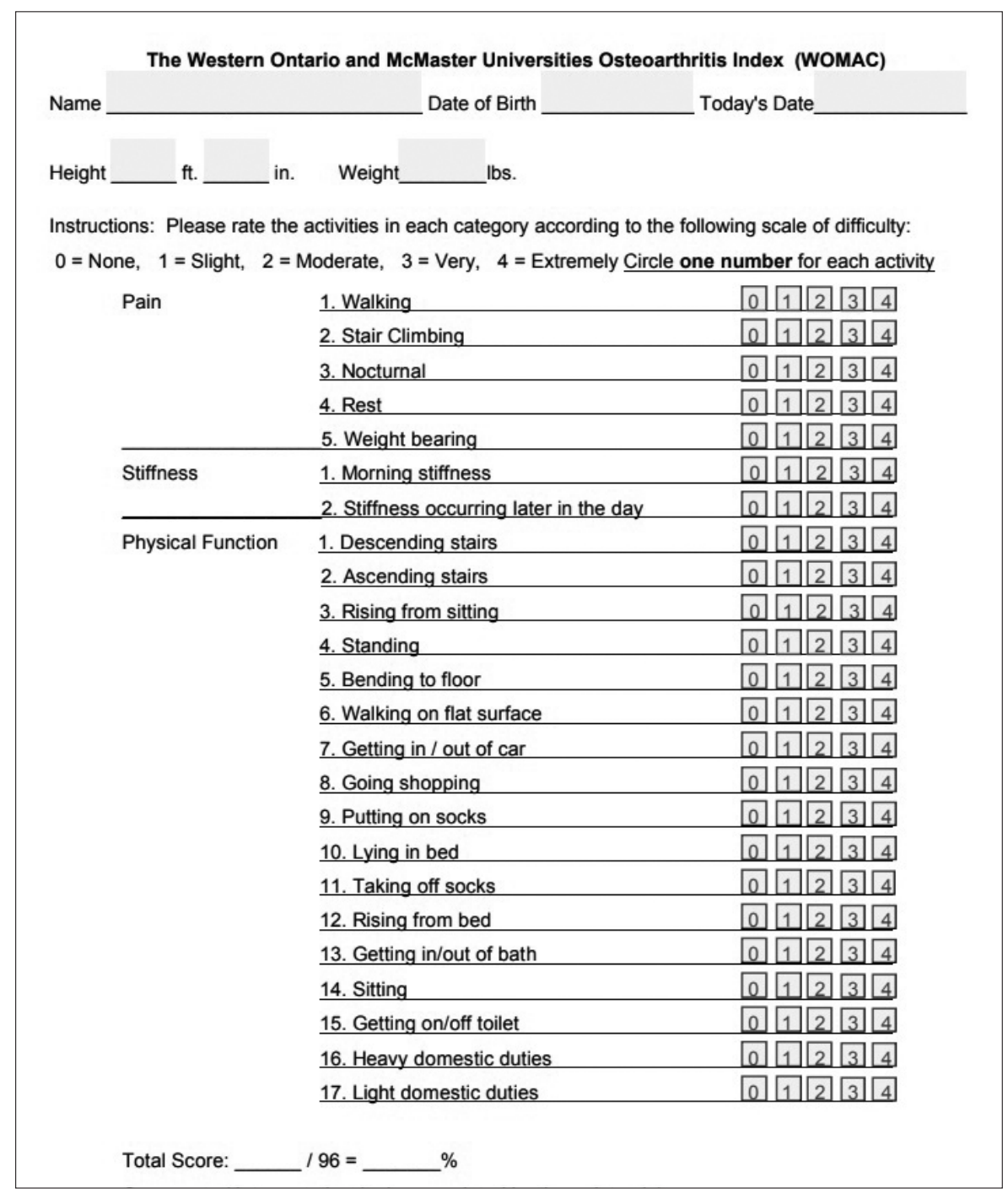

The WOMAC is a self-administered questionnaire that is composed of 24 questions categorized into three subscales (pain, stiffness, and physical function). 
Seihee Yoon, et al.

Appendix 2. The 2011 Knee Society Scores (KSS)

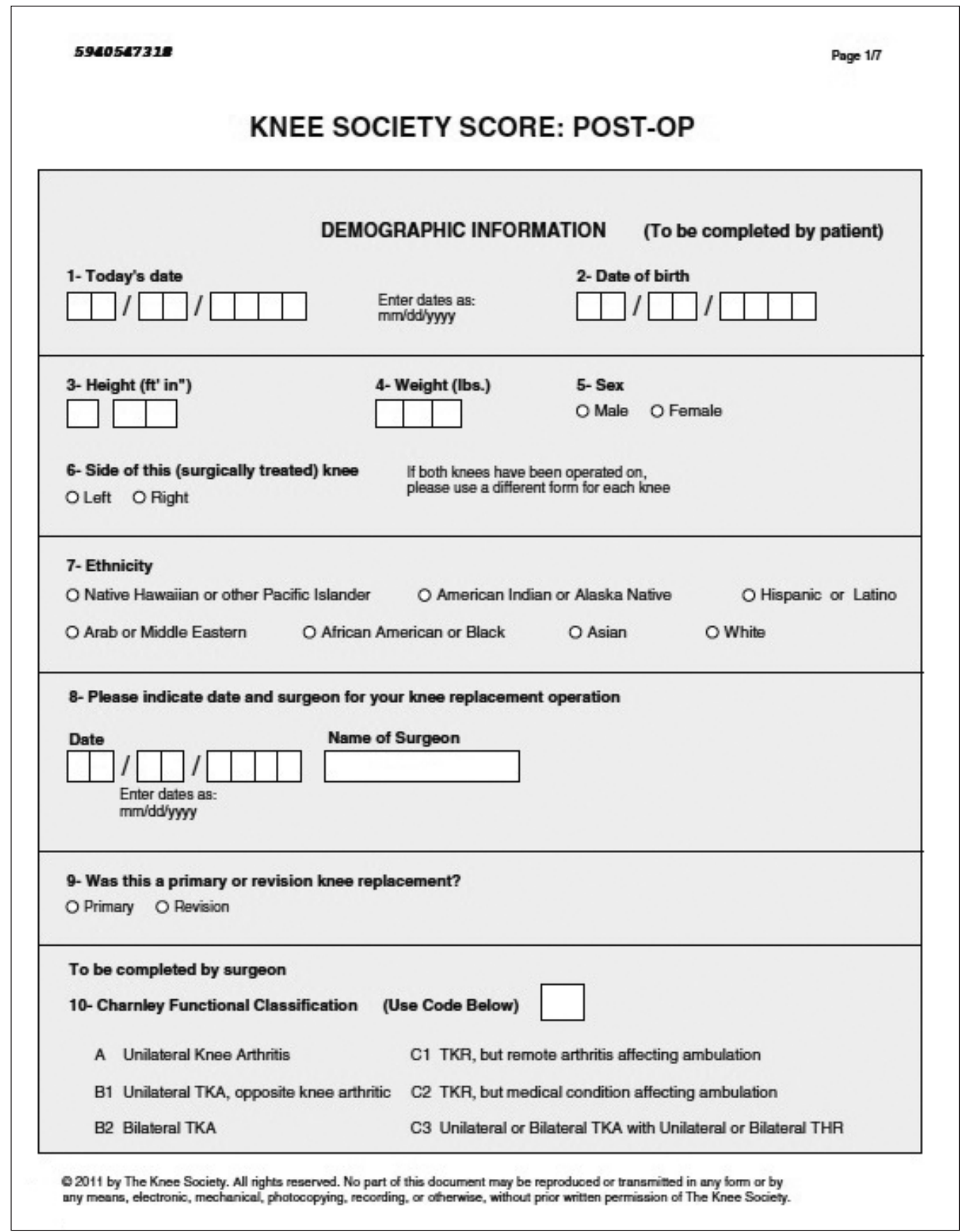

The 2011 KSS consists of four subscales: (A) objective knee scores (7 items, 100 points); (B) symptom scores ( 3 items, 25 points); (C) patient satisfaction scores (5 items, 40 points); and (D) functional activity scores (19 items, 100 points). 